\title{
PEEK Physical Surface Modification: Evaluation Of Particles Leaching Process
}

\author{
Flavia Suzany Ferreira dos Santos ${ }^{\circledR}$, Gabriel Goetten de Lima ${ }^{b}$, Rita de Cássia Alves Leal Cruz ${ }^{a}$,

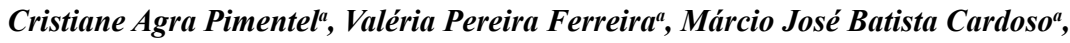 \\ Rodrigo Alvarenga Rezende ${ }^{c}$, Marcus Vinícius Lia Fook \\ ${ }^{a}$ Departamento de Engenharia de Materais, Universidade Federal de Campina Grande, 58429-900, \\ Campina Grande, PB, Brasil \\ ${ }^{b}$ Materials Research Insitute, Athlone Institute of Thechnology, Athlone, Ireland \\ ${ }^{c}$ Centro de Tecnologia da Informação Renato Archer, 13069-901, Campinas, SP, Brasil
}

Received: July 24, 2018; Revised: December 03, 2018; Accepted: January 17, 2019

\begin{abstract}
Polyetheretherketone (PEEK) has been prominent in orthopedic implants; however, it is inert, preventing interaction between the implant and adjacent bone tissues. One way to overcome this characteristic is physical modification its on surface by particle leaching promoting greater osseointegration. The objective of this research was to develop and characterize the PEEK using a surface modification technique via particle leaching. For of the samples, a layer deposition of $\mathrm{NaCl}$ was used on PEEK. This was subjected to the pressure of $850 \mathrm{~kg} / \mathrm{cm} 2$ by $390^{\circ} \mathrm{C}$ for 20 minutes. After cooling, were subjected to leaching process particles. The results indicated a porous surface exhibiting uniform and homogeneous morphology with defined pores interconected, to in the range of 140-373 $\mu \mathrm{m}$, with an average diameter of $273 \mu \mathrm{m}$. These evidenced the considerable $\mathrm{NaCl}$ removal after the leaching process, with cavities from ideal sizes which promotes adequate cellular accommodation and distinct roughness, giving an overall possibility of being able to obtain a material more able to receive the cells while also possibly presenting cell viability. Although the compressive strength presented low values, it can still be suitable for applications in areas with a reduced modulus of elasticity.
\end{abstract}

Keywords: Surface modification, PEEK, NaCl particles leaching, polymer.

\section{Introduction}

Poly (ether ether ketone) - PEEK has been outstanding in the use of orthopedic implants. Coincidently, the availability of this polymer match with the growing interest in development of fracture fixation rods and plaques. PEEK eventually presented widespread relevance due to their high resistance, biocompatibility and mechanical properties similar to human bones $^{1}$. When compared to traditional metal implants, the mechanical properties of PEEK reduces the effect of bone atrophy - stress shielding ${ }^{2}$. PEEK materials also exhibit radiolucent characteristics, implants manufactured from PEEK are compatible with imaging diagnoses, unlike metal implants ${ }^{3}$. Its heat resistance and chemical compatibility with various sterilization techniques, coupled with low cost molding techniques, led PEEK to be a practical and economical material for the manufacturing of medical devices ${ }^{4}$.

PEEK has several advantages and characteristics; however, it is biologically inert, which prevents the interaction between the implant and adjacent bone tissues. Without osseointegration (implant-to-bone interaction), the implants may be loosen or wear, causing pain, inflammation or even serious risk injuries deformity to the patient. For these reasons, several approaches have been focused to overcome the inert character

*e-mail: flaviasuzanyfs@gmail.com of $\mathrm{PEEK}^{5,6}$ An alternative is the physical modification of its surface by particles leaching.

Surface modification alters the surface characteristics of a material for a specific application without affecting the mass properties. Idealistically, surface modifications should offer many advantages such as controlment on porosity, improvements in wettability, mechanical properties and biocompability ${ }^{7}$.

This modification would be beneficial the applications and studys area that require a greater interaction between bone and implant since the surface modification in permanent, porous structures have the ability to provide a transitional space between bone and a biomaterial substrate (which provides the main structural support) and an appropriate level and geometry of porosity will enable bone in-growth and hence enhanced integration between the bone and the biomaterial structure $^{8}$. Surface porous also plays a major role as it can affect the long-termmechanical stability and early fixation through mechanicalinterlocking between mineralised bone and the porous surface. It also helps with the adsorption of proteins and theadhesion of osteoblastic cells, thus increasing the rate of osseoin-tegration ${ }^{9}$.

The physical modification comprises the physical change of the material structure and has the advantage of restricting porosity only on the surface of the polymer, thus promoting osseointegration ${ }^{10}$, not altering the hydrophilic or hydrophobic 
character of this material, being considered important factors for applications in the medical area.

A simple yet very useful technique is the particle leaching method. This method involves the selective leaching of a mineral, usually the $\mathrm{NaCl}$ salt or an organic compound such as sucrose, to generate a porous structure. These salt particles are either incorporated into the polymer in solution or moulded together during melting by techniques such as compression, extrusion or injection. The mechanism of leaching $\mathrm{NaCl}$ crystals consists on solubilization of porogenic agents in water, through a mechanical drag of these particles ${ }^{11}$. Recent works in literature have shown it is also possible with leaching method to produce porous structure which mimics the layered cranial bone a three layered structure composed of compact bones and a porous in the middle.

Due to the adhesive weak properties of raw PEEK polymers for biomedical applications, the objective of this work was to develop and characterize PEEK with the particle leaching technique, as well as to evaluate the influence caused in the morphology, mechanical and biological behavior of the samples obtained.

\section{Materials}

For the production of the specimens, the PEEK Vicote 702 powder polymer supplied by Victrex ${ }^{\circledR}$ was used, whith particle size of 10 to $50 \mu \mathrm{m}$. Sodium chloride $(\mathrm{NaCl})$ P.A was purchased by the Nuclear brand, whith molecular weight 55.44 and $99.8 \%$ purity content.

\subsection{Experimental procedure}

The PEEK polymer was oven dried at $150^{\circ} \mathrm{C}$ for $3 \mathrm{~h}$. For the preparation of the specimens, a $30 \mathrm{~mm}$ diameter and 10 mm thick cylindrical metal mould containing approximately one layer $1 \mathrm{~g}$ of $\mathrm{NaCl}$ followed by the addition of another layer containing $2 \mathrm{~g}$ of PEEK was used. After this procedure, the mold filled with $\mathrm{NaCl}$ and PEEK was submitted to hydraulic press and subjected to a pressure of $850 \mathrm{~kg} / \mathrm{cm} 2$ and synthesized at $390{ }^{\circ} \mathrm{C}$ for 20 minutes. After cooling done at room temperature, these samples were subjected to the particulate leaching process, samples were immersed in water for the removal of $\mathrm{NaCl}$ at $72 \mathrm{~h}$, with subsequently immersion in water under magnetic stirring for 60 minutes at $100{ }^{\circ} \mathrm{C}$, to produce modification of the surface of this polymer. After this procedure, these samples were dried in an oven at $60{ }^{\circ} \mathrm{C}$ for 30 minutes.

\subsection{Characterization of samples}

\subsubsection{Scanning Electron Microscopy (SEM)}

SEM was used for the evaluation of the rough surface morphology and the cross section of the samples using a Hitachi model TM-1000 electronic scanning electron microscope, with maximum magnification of $10000 \mathrm{x}$, depth of focus of $1 \mathrm{~mm}$, resolution of $30 \mathrm{~nm}, 15 \mathrm{KV}$, low vacuum and varied pressure ( 1 to $270 \mathrm{~Pa}$ ), without metallic coating. For the application of this technique, increases of $100 \mathrm{x}$ and 200x were used. For the evaluation of cross-sectional morphology, the samples were fractured in liquid nitrogen.

\subsubsection{Cavity size distribution and particles}

In this technique, the ImageJ software was used to perform the measurements of the medium diameter of 80 $\mathrm{NaCl}$ particles and medium diameter of 80 cavities of the surface PEEK.

\subsubsection{Citotoxicity}

Before the test, the samples were sterilization in an autoclave (Autoclave Vertical CS, Prismatec) for 30 minutes to prevent any any external contamination. As well as, the test was performed in triplicate to ensure greater reliability of the results.

A cytotoxicity assay was performed according to ISO 10993-5:2009: Biological evaluation of medical devices-Part 5 : Tests for in vitro cytotoxicity ${ }^{12}$. To evaluate cytotoxicity, the method of direct contact between the substrate of the material and the cells was used. Was used The L929 fibroblast cell line (ATCC ${ }^{\circledR}$ NCTC clone 929) was grown in an RPMI culture medium (RPMI 1640 Medium, Gibco $^{\circledR}$ - Invitrogen Corporation, Grad Island, USA) supplemented with 10\% bovine foetal serum (Gibco ${ }^{\circledR}$, Life Technologies) and 1\% antibiotic-antimycotic solution (Gibco ${ }^{\circledR}$, Life Technologies). A concentration of $1 \times 10^{5}$ cells $/ \mathrm{mL}$ was used in RPMI culture medium and $100 \mu \mathrm{L}$ were added to a 96 well plate. The plate was transferred to a $\mathrm{CO}_{2}$ incubator at $37^{\circ} \mathrm{C}$ for 24 hours. Afterwards, the culture medium was then aspirated from all wells, and added more $200 \mu \mathrm{L}$ of RPMI 1640 culture medium the samples. These plates were incubated again in a $\mathrm{CO}_{2}$ incubator at $37^{\circ} \mathrm{C}$ for a further $24 \mathrm{hrs}$, totaling 48 hours from the beginning of the assay. After these 48 hours these samples and the RPMI culture medium were removed and $100 \mu \mathrm{l}$ of MTT (1mg / ml) (3- (4,5-dimethyl-2-azol-2yl)-2,5-diphenyltetrazolium bromide) were added in PBS (Phosphate Buffered Saline). Finally, these plates were incubated in a $\mathrm{CO}_{2}$ oven at $37^{\circ} \mathrm{C}$ for $3 \mathrm{hrs}$. The supernatant was discarded and $100 \mu \mathrm{L}$ of isopropyl alcohol were added. Optical density reading was determined on a microplate reader (Victor3 - Perkin Elmer) at $570 \mathrm{~nm}$ with $650 \mathrm{~nm}$ reference filter. Cell viability was calculated as a percentage by the modified z-score test for detecting the outliers. According to the material cytotoxicity classification of ISO 10993-5 2013 , cell viability (\%) determines the cytotoxicity of the material so that below $70 \%$, the material is considered potentially cytotoxic.

\subsubsection{Compressive strength}

The compressive strength tests were performed according to ASTM D695-15 ${ }^{13}$, and its purpose was to evaluate the 
modulus of elasticity as well as changes in the compressive strength of the sample with the modified surface. These tests were performed on Instron hydraulic universal testing machine (Model 3366) with a load cell of $10 \mathrm{kN}$ and a velocity of $1.3 \mathrm{~mm} / \mathrm{min}$. The modulus of elasticity in the elastic region was calculated according to the calculation of the tangent of the curve and tension at $10 \%$ strain were observed.

\subsubsection{Atomic Force Microscopy (AFM)}

AFM analyzes were performed using a NEXT Solver microscope, model NT-MDT. The baffle tip operated in contact mode, at a temperature of $22{ }^{\circ} \mathrm{C}$ and humidity of $45 \%$. The analysis was performed in the sample on its smooth and rough surface (obtained after leaching of particles) for possible comparison.

\section{Results}

\subsection{Scanning Electron Microscopy (SEM)}

Figure 1 exhibits the representative image of the PEEK surface with the surface modification, without magnification (a), Figure 1 (b) smooth without pores with magnification of 100x, surface porous with magnification of 100x (c) and with magnification 200x (d)respectively.

Analyzing Figure 1(c), a uniform and homogeneous morphology can be observed on the rough surface, with welldefined cavities and thin walls interconnecting one cavity to another. It is known that the compressive molding technique with $\mathrm{PEEK}$ and $\mathrm{NaCl}$ promotes a porous morphology after removing the salt in the process leaching; and qualitatively analyzing the images (c) and (d) of the morphology samples, it can be deduced a very well porosity distribution and small variations on its diameter.

The samples prepared have relatively homogeneous pore structure throughout the structure with poros an average diameter of $273 \mu \mathrm{m}$. The proposed process allows a better interconnection between the pores left by the salt removal in the leaching process, as can be seen more clearly in Figure 1(d) and a interconnected porous structure resulting from the selective polymer leaching. This good interconnectivity between the pores is strongly desired in tissue engineering, as the exchange of nutrients and cell waste would be improved ${ }^{11}$.

Figure 2 exhibits the representative image of the cross section of the PEEK with the physical surface modification (a) and Figure 2 (b) and (c) the micrographs of that cross section with magnification of 100 and 200x respectively.

With respect to cross section micrographs (Figure 2(b) and (c)), two distinct regions can be noticed, one region exhibits a morphology with small roughness, dense and smooth. This region characterizes as the raw PEEK polymer since limited salt crystals could have diffused towards the
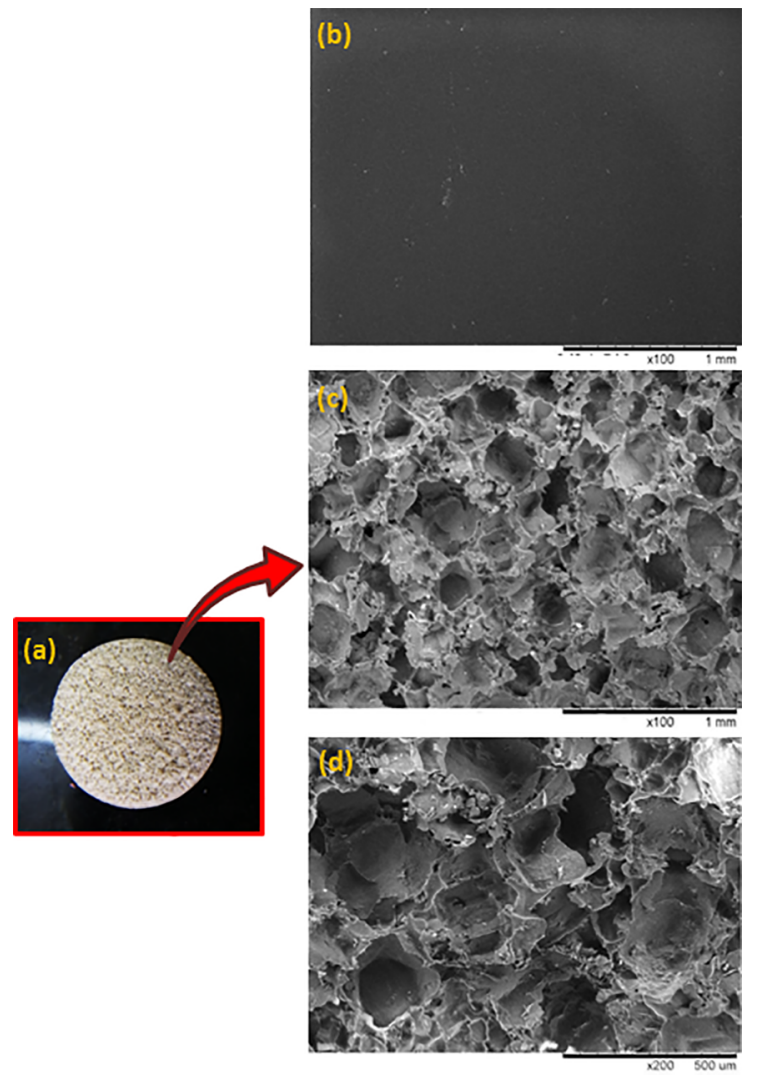

Figure 1. Micrographs of the porous surface of the sample without magnification (a), surface without pores with magnification of $100 x(b)$, surface porous with magnification of 100x (c) and with magnification 200x (d).

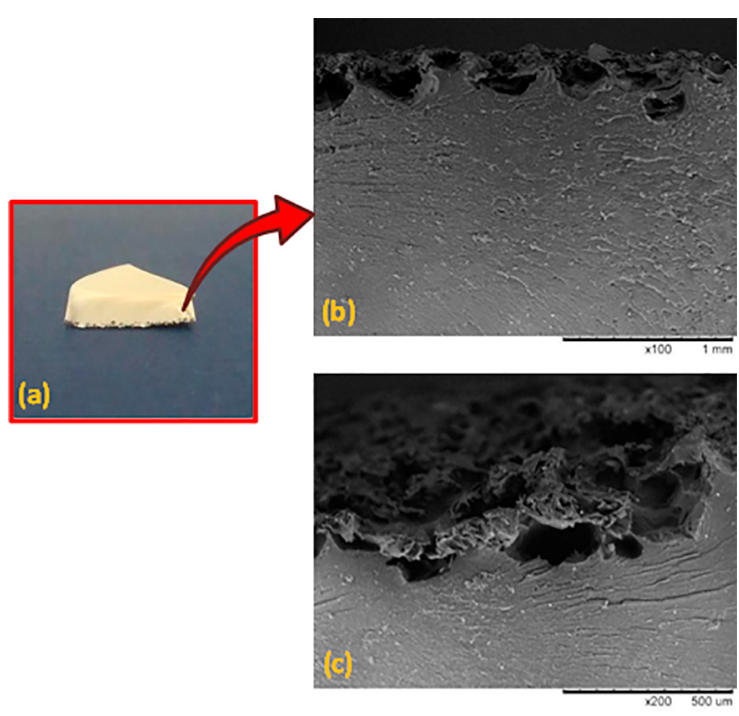

Figure 2. Micrographs of the cross section of the sample, observed without magnification (a), with magnification of 100 (b) and 200x (c).

interior part of it. Contrary, the surface region exhibits a roughness profile and porous with uniform morphology, this occurs due to the leaching effect of the salt crystals on the surface of this polymer. 
Also, the absence of remaining salt crystals suggests that the dissolution of the salt crystals is completed and that the pores are well interconnected ${ }^{11}$.

The pore space characteristics offered by the salt template are unique and different from other scaffold manufacturing processes. Sintering salt particles has produced an interconnected spherical pore network in the structure ${ }^{14}$.

\subsection{Cavity size distribution and particles}

The leaching of $\mathrm{NaCl}$ powders leads to solubilization of porogenic agent in water, through a mechanical drag.
The solubilization of this porogenic agent will modified the morphology of these materials, resulting in a mean porous diameter related to the $\mathrm{NaCl}$ granulometry.

The particle size of the salt crystals is in the range of 185-379 $\mu \mathrm{m}$, with a mean particle size of $276 \mu \mathrm{m}$, and according to the granulometric distribution performed through the histogram (Figure 3.a), about $64 \%$ of the $\mathrm{NaCl}$ particles are in the range of 201-300 $\mu \mathrm{m}$.

According to the mean diameter values of the porosity, it was possible to observe a pores size in the range of 140$373 \mu \mathrm{m}$, with an average diameter of $273 \mu \mathrm{m}$ (Figure 3.b).
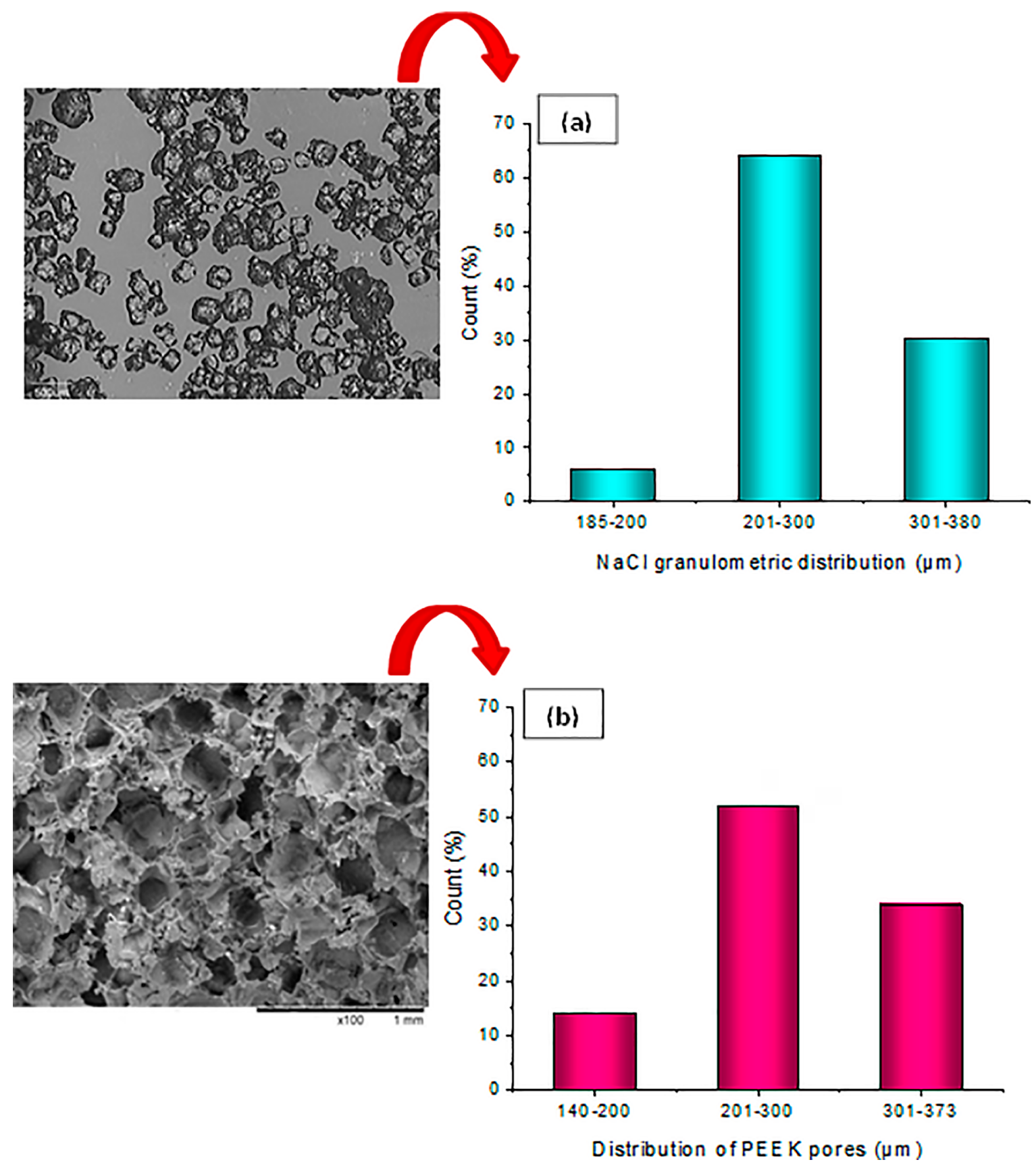

Figure 3. Histograms of $\mathrm{NaCl}$ granulometric distribution (a) and distribution of PEEK porous (b) measured with ImageJ software. 
In relation to the pores diameter, there was a count decrease that can be related to the $\mathrm{NaCl}$ particles disrupting in the compression procedure, causing a small decrease of the pores after the particle leaching process. However, analyzing the histogram shown in Figure 3.a and 3.b, it was possible to perceive that the porosity distribution follows a correlation with the granulometric distribution of the salt crystals with a decrease in the mean region, though the major region still presents the highest factor of porosity with $52 \%$. This porosity distribution based on the salt leaching evidences the controlled mechanism that was possible to obtain.

The results evidenced in the present study with respect to the mean size of the porous are in agreement with the average pore size range estimated by ${ }^{8}$ that used the compression technique and the $\mathrm{NaCl}$ particle leaching method for the production of PEEK porous structures, with results of $85 \%$ porosity and average pore diameter estimated between 250 and $350 \mu \mathrm{m}$. A few authors investigated a new method to obtain a PEEK based material with porous surface to promote osseointegration, using the $\mathrm{NaCl}$ particle leaching compression technique and achieved results with average pore size of $280 \mu \mathrm{m}^{10}$. And ${ }^{15}$ observed the effect of pore size on tissue regeneration. The $5 \mu \mathrm{m}$ pores allow neovascularization, 5-15 $\mu \mathrm{m}$ fibroblast growth, besides 40-100 $\mu \mathrm{m}$ osteoid matrix growth and 100-350 $\mu \mathrm{m}$ bone regeneration.

\subsection{Compressive strength}

It can be observed in the stress-strain graph (Figure 4) that the initial phase is linear. The modulus of elasticity is numerically equal to the value of the $\mathrm{r}^{2} \pm 0,9984$ linearization curve. Therefore it was possible, from the linear elastic region, to obtain the mean modulus of elasticity which is 54.73 MPa (Figure 4.b).

The small linear elastic region, observed in the initial compression stage, presented low tensile strength due to flexion of the walls in the cavities - increasing the porosity in the samples can lead to lower values of its strength.

From $3 \%$ of strain, the graph presented a region of a well-defined plateau in the curve (Figure 4.a), which might have occurred due to where the compaction of the porous starts. This region corresponds to a higher energy absorption, in which the room occupied by the porous are filled by the compressed material ${ }^{16,17}$.

Comparing the elastic ( $\mathrm{E}=54,73 \mathrm{MPa}$ ) of the investigated samples (Figure 4.b) with the theoretical elastic modulus of PEEK (3,700 MPa) and human bone (20-500 MPa trabecular, and $12,800-17,700$ cortical MPa), it was observed that the sample had a lower elastic modulus than reported in literature. Regarding bone, the value found in this study for PEEK is within the elastic modulus value of the trabecular bone (20$500 \mathrm{MPa})^{1,18}$. The reason for this could be due to the porous structure which affects the youngs modulus of the material, as discussed already and reported by several authors.

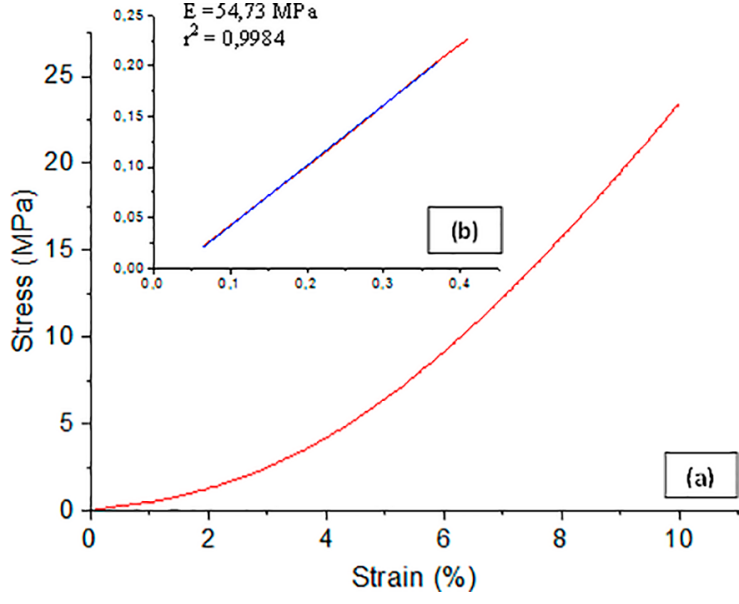

Figure 4. Voltage-strain curve for sample tested.

In relation to the tension at $10 \%$ deformation (compressive strength) in the literature, the values of $125 \mathrm{MPa}$ of dense PEEK $^{19}, 1.5-38 \mathrm{MPa}$ for trabecular bone and 88-190 MPa for bone were observed cortical ${ }^{20,21}$. In the results presented in this article, the value of $23.78 \pm 0.31 \mathrm{MPa}$ was observed, which represents a reduction in the compressive strength of approximately $84 \%$ in relation to the dense PEEK. However, this value was shown to be comprised between the values of compressive strength of the trabecular bone. Similar values were found by Bakar et $\mathrm{al}^{22}$, the authors observed that there was a reduction of up to $86 \%$ in the compressive strength of the material, due to the high porosity and the relatively weak bonds created during the melting of the polymer ${ }^{20,23,24}$.

Siddiq and colleagues ${ }^{8}$ used the particle leaching method for the production of PEEK porous structures. This resulted in structures with excellent repeatability, homogeneity and uniformity in the pores. However, with respect to the compression test, these porous structures showed lower modulus and compressive strength results than the trabecular bone. Therefore, it can be concluded that the structure presented is more feasible for applications where they do not require load and reduced modulus. Converse et $\mathrm{al}^{25}$ evaluated the mechanical properties of hydroxyapatite reinforced polyetherketoneketone composite scaffolds in order to investigate the effects of porosity, hydroxyapatite concentration and mold temperature. These variables revealed that the increase in porosity reduced the elastic modulus, besides allowing the mechanical properties to be adapted to imitate the human trabecular bone, with satisfactory results and a modulus of elasticity superior to $50 \mathrm{MPa}$, similar to that of the human trabecular bone.

Therefore, the modification of the PEEK surface caused some changes in the mechanical properties of the tested sample, such as the decrease of the elastic modulus, and the compressive strength. This decrease in the resistance, as well as the decrease in the value of the elastic modulus when compared to the dense PEEK was already expected, 
since, the presence of the pores on the surface caused the compressive load to be dissipated in a smoother way, reducing the resistance and the module. However, although with some limitations these results were satisfactory, since they are within the results of modulus (20-500 MPa) and compressive strength (1,5-38 MPa) of the trabecular bone when compared to those found in the literature. Although the compressive strength of these porous surface materials is low, it may still be suitable for applications with a reduced modulus $^{26}$

\subsection{Atomic Force Microscopy}

Figure 5 exhibits the AFM of the rough and smooth surface of the PEEK obtained by processing through compression moulding followed by the leaching of particles in an area of $1 \mu \mathrm{m}^{2}$.

It was possible to observe that surfaces of the polymeric samples presented an irregular topography formed by parallel lines with a height of $1.11 \mu \mathrm{m}$ on the rough surface and 53 $\mathrm{nm}$ on the smooth surface. Changes in brightness indicate differences in height, ie the brightest regions have the maximum height and the darkest regions have the lowest, which might have been formed by the leaching process of particles of porogenic $\mathrm{NaCl}$ in water

From the AFM results the arithmetic mean roughness (Ra) and the mean square roughness (Rms) of the rough and smooth PEEK surfaces was measured, respectively (89.09 and $109.48 \mathrm{~nm}$ ) and (5.809 and $7.366 \mathrm{~nm})$. This information confirms that the use of $\mathrm{NaCl}$ with PEEK forms a distinct surface when compared to the processing of PEEK without $\mathrm{NaCl}$, presenting a rough surface formed due to solubilization of the porogenic agent in water.

Aparecida and colleagues ${ }^{27}$, cite that roughness created in the material by means of the compression technique followed by leaching of particles is important due to the possibility of creating a more propitious material which can shelter cells, improving their use as porous biomaterial for replacement and bone regeneration. Almasi et $\mathrm{al}^{9}$ also used the AFM technique to measure the surface roughness of surface modified PEEK samples and observed that increasing surface roughness could increase cell attachment through mechanical blockage between mineralized bone and implant.

\subsection{Cytotoxicity}

The samples had an average value of $86 \%$ of cell viability whith standard deviation of \pm 11 based on the calculation of uncertainty and z-score; therefore, possibility it is not cytotoxic, once the cells were preserved being the possibility biocompatible sample with a value above that provided by ISO 10993-5 2009 ${ }^{12}$, which is at least $70 \%$. In addition the samples gave an indication for cell proliferation, so the method was effective, since it can promote a better osseointegration. This result corroborates with the ones found in literature, Li et $\mathrm{al}^{28}$ found that PVA-PEEK hydrogels presented a value of $90 \%$ cell viability and revealed that hydrogels have no negative effect on viability and cell proliferation indicating a good biocompatibility. Others authors studying the preparation of PEEK composites for bone fixation, used the MTT method to measure the cellular viability of their structures and exhibiting good cellular compatibility ${ }^{29}$. Sheiko et $\mathrm{al}^{30}$ performed a nitinol wire coated with PEEK work for applications as biomaterial and in their observations on cell biocompatibility of Nitinol coated with PEEK exhibited excellent biocompatibility in L929 fibroblast cells.

\section{Conclusion}

The results presented in this study exhibited that there was an effective modification in the surface of the PEEK by the technique of compression, thermal treatment followed by leaching of salt particles, thus obtaining structures with modified surface. The rough surface of the sample exhibited an uniform and homogeneous morphology, with well-defined pores and thin walls interconnecting one pore to another. In addition, the methodology used leads to the conformation and preservation of the pores presented on the modified surface, as a consequence of the polymer fusion and effective leaching of the particles. The material obtained showed considerable $\mathrm{NaCl}$ removal in the particle leaching process, with porosity of ideal sizes to promote adequate cellular accommodation
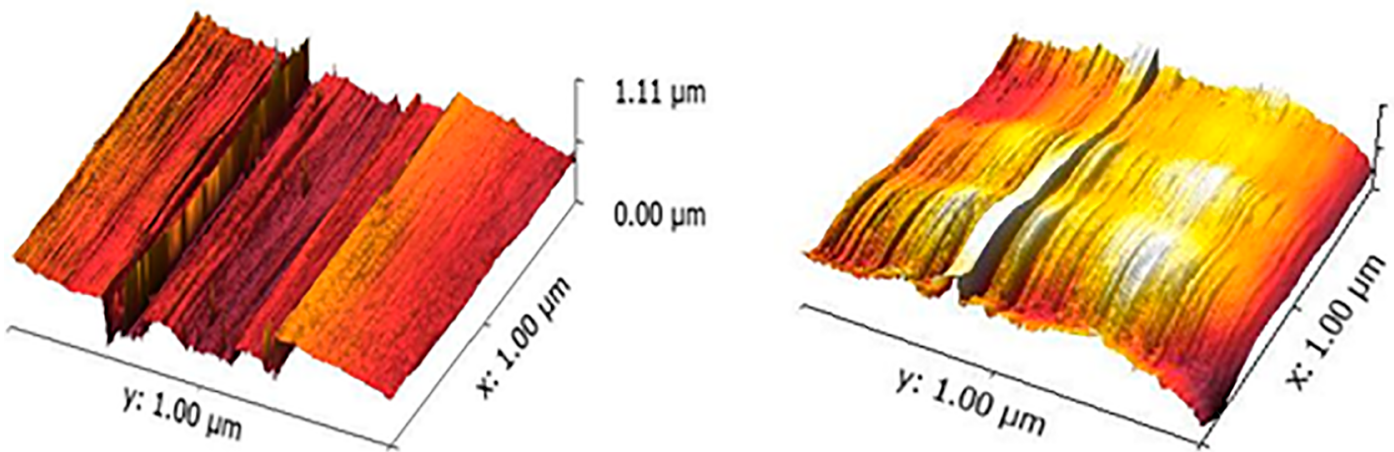

$53 \mathrm{~nm}$

$0 \mathrm{~nm}$

Figure 5. Atomic force microscopy of the PEEK porous (a) and smooth (b) surface, respectively. 
as well as a distinct rough surface offering the possibility of creating a material more conducive to receive the cells besides presenting viability indicative above the standard ISO 10993-5 2009. The compressive strength results demonstrated the difficulty in maintaining the mechanical properties of this material. However, although the compressive strength of the sample presented low values, it may still be suitable for applications in areas with a reduced modulus of elasticity, thus corroborating results in literature.

\section{Acknowledgments}

The authors are grateful to CAPES.

\section{References}

1. Kurtz SM, ed. PEEK Biomaterials Handbook. Oxford: William Andrew; 2011.

2. Niinomi M, Nakai M. Titanium-Based Biomaterials for Preventing Stress Shielding between Implant Devices and Bone. International Journal of Biomaterials. 2011;2011:836587.

3. Toth JM, Wang M, Estes BT, Scifert JL, Seim HB III, Turner AS. Polyetheretherketone as a biomaterial for spinal applications. Biomaterials. 2006;27(3):324-334.

4. Durham JW III, Rabiei A. Deposition, heat treatment and characterization of two layer bioactive coatings on cylindrical PEEK. Surface and Coatings Technology. 2016;301:106-113.

5. Ma R, Tang T. Current strategies to improve the bioactivity of PEEK. International Journal of Molecular Sciences. 2014;15(4):5426-5445.

6. Deng Y, Liu X, Xu A, Wang L, Luo Z, Zheng Y, et al. Effect of surface roughness on osteogenesis in vitro and osseointegration in vivo of carbon fiber-reinforced polyetheretherketonenanohydroxyapatite composite. International Journal of Nanomedicine. 2015;10:1425-1447.

7. Jasso-Gastinel CF, Kenny JM, eds. Modification of Polymer Properties. Oxford: William Andrew; 2017.

8. Siddiq AR, Kennedy AR. Porous poly-ether ether ketone (PEEK) manufactured by a novel powder route using near-spherical salt bead porogens: characterisation and mechanical properties. Materials Science \& Engineering: C. 2015;47:180-188.

9. Almasi D, Izman S, Assadian M, Ghanbari M, Abdul Kadir MR. Crystalline ha coating on peek via chemical deposition. Applied Surface Science. 2014;314:1034-1040.

10. Evans NT, Torstrick FB, Lee CS, Dupont KM, Safranski DL, Chang WA, et al. High-strength, surface-porous polyether-etherketone for load-bearing orthopedic implants. Acta Biomaterialia. 2015;13:159-167.

11. Reignier J, Huneault MA. Preparation of interconnected poly(ecaprolactone) porous scaffolds by a combination of polymer and salt particulate leaching. Polymer. 2006;47(13):4703-4717.
12. International Organization for Standardization - ISO. ISO 10993-5:2009 - Biological evaluation of medical devices - Part 5: Tests for in vitro cytotoxicity. Geneva: ISO; 2009.

13. ASTM International. ASTM D695-15 - Standard Test Method for Compressive Properties of Rigid Plastics. West Conshohocken: ASTM International; 2015.

14. Gross KA, Rodriguez-Lorenzo LM. Biodegradable composite scaffolds with an interconnected spherical network for bone tissue engineering. Biomaterials. 2004;25(20):4955-4962.

15. Wang H, Zhang S, Wang G, Yang S, Zhu Y. Tribological behaviors of hierarchical porous PEEK composites with mesoporous titanium oxide whisker. Wear. 2013;297(1-2):736-741.

16. Ashby MF. The properties of foams and lattices. Philosophical Transactions of the Royal Society A: Mathematical, Physical and Engineering Sciences. 2006;364(1838):15-30.

17. Gibson LJ. Biomechanics of cellular solids. Journal of Biomechanics. 2005;38(3):377-399.

18. Belabarba C, Mirza SK, Chapman JR. Biomateriais e suas Aplicações na Cirurgia da Coluna. In: Vaccaro AR, Kim H, Henn H. Cirurgia da Coluna Princípios e Prática. Rio de Janeiro: DiLivros; 2007. p. 35-65.

19. Victrex. PEEK. Lancashire: Victrex; 2015. Available from: $<\mathrm{http}: /$ www.victrex.com>. Access in: 5/9/2018.

20. Smith WF, Hashemi J. Fundamentos de Engenharia e Ciencia dos Materiais. Porto Alegre: AMGH Editora; 2012.

21. Nabais CR. Análise Numérica da Interface Osso-Cimento na Artroplastia da Anca. [Dissertação]. Porto: Faculdade de Engenharia da Universidade do Porto; 2006.

22. Abu Bakar MS, Cheng MHW, Tang SM, Yu SC, Liao K, Tan CT, et al. Tensile properties, tension-tension fatigue and biological response of polyetheretherketone-hydroxyapatite composites for load-bearing orthopedic implants. Biomaterials. 2003;24(13):22452250 .

23. Converse GL, Yue W, Roeder RK. Processing and tensile properties of hydroxyapatite-whisker-reinforced polyetheretherketone. Biomaterials. 2007;28(6):927-935.

24. Landy BC, Vangordon SB, McFetridge PS, Sikavitsas VI, JarmanSmith M. Mechanical and in vitro investigation of a porous PEEK foam for medical device implants. Journal of Applied Biomaterials \& Functional Materials. 2013;11(1):e35-44.

25. Converse GL, Conrad TL, Roeder RK. Mechanical properties of hydroxyapatite whisker reinforced polyetherketoneketone composite scaffolds. Journal of the Mechanical Behavior of Biomedical Materials. 2009;2(6):627-635.

26. Jarman-Smith M, Brady M, Kurtz SM, Cordaro NM, Walsh WR. Porosity in polyaryletheretherketone. In: Kurtz SM, ed. PEEK Biomaterials Handbook. Oxford: William Andrew; 2011. p. 181199. 
27. Aparecida AH, Guastaldi AC, Fook MVL. Desenvolvimento e caracterização de suportes porosos de polietileno de ultra alto peso molecular (PEUAPM) para utilização como biomaterial para reposição e regeneração óssea. Polímeros. 2008;18(4):277-280.

28. Li W, Kang J, Yuan Y, Xiao F, Yao H, Liu S, et al. Preparation and characterization of PVA-PEEK/PVA-ß-TCP bilayered hydrogels for articular cartilage tissue repair. Composites Science and Technology. 2016;128:58-64.
29. Luo H, Xiong G, Yang Z, Raman SR, Li Q, Ma C, et al. Preparation of three-dimensional braided carbon fiber-reinforced PEEK composites for potential load-bearing bone fixations. Part I. Mechanical properties and cytocompatibility. Journal of the Mechanical Behavior of Biomedical Materials. 2014;29:103-113.

30. Sheiko N, Kékicheff P, Marie P, Schmutz M, Jacomine L, Perrin-Schmitt F. PEEK (polyether-ether-ketone)-coated nitinol wire: Film stability for biocompatibility applications. Applied Surface Science. 2016;389:651-665. 